\title{
Single-cell and spatially resolved analysis uncovers cell heterogeneity of breast cancer
}

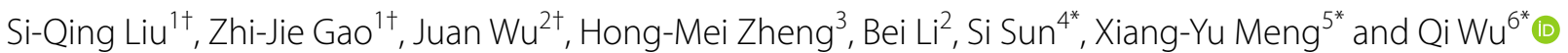

\begin{abstract}
The heterogeneity and the complex cellular architecture have a crucial effect on breast cancer progression and response to treatment. However, deciphering the neoplastic subtypes and their spatial organization is still challenging. Here, we combine single-nucleus RNA sequencing (snRNA-seq) with a microarray-based spatial transcriptomics (ST) to identify cell populations and their spatial distribution in breast cancer tissues. Malignant cells are clustered into distinct subpopulations. These cell clusters not only have diverse features, origins and functions, but also emerge to the crosstalk within subtypes. Furthermore, we find that these subclusters are mapped in distinct tissue regions, where discrepant enrichment of stromal cell types are observed. We also inferred the abundance of these tumorous subpopulations by deconvolution of large breast cancer RNA-seq cohorts, revealing differential association with patient survival and therapeutic response. Our study provides a novel insight for the cellular architecture of breast cancer and potential therapeutic strategies.
\end{abstract}

Keywords: Single-nucleus RNA sequencing, Spatial transcriptomics, Breast cancer, Tissue architecture, Heterogeneity

To the editor,

A universal feature of breast cancer $(\mathrm{BC})$ is the complex cellular ecosystems, whereby intra- and inter-tumor heterogeneity is crucial for determining malignant progression and response to treatment. In this study, we aimed to decipher the heterogeneity and complex architecture of breast cancer by combining two complementary highresolution omics tools $[1,2]$.

We analyzed 2 primary tumors (BC-A and BC-B) by single-nucleus RNA sequencing (snRNA-seq, $10 \times$ genomics) $[2,3]$. Fresh samples from $\mathrm{BC}-\mathrm{A}$ were

\footnotetext{
*Correspondence: 59333173@qq.com; mengxy_whu@163.com; waiwai@whu.edu.cn

${ }^{\dagger}$ Si-Qing Liu, Zhi-Jie Gao and Juan Wu have contributed equally to this work.

${ }^{4}$ Department of Clinical Laboratory, Renmin Hospital of Wuhan University, Hubei Province, 238 Ziyang Road, Wuhan 430060, People's Republic of China

${ }^{5}$ Center for Single-Cell Omics and Tumor Liquid Biopsy, Zhongnan Hospital of Wuhan University, Wuhan, Hubei, People's Republic of China

${ }^{6}$ Tongji University Cancer Center, Tenth People's Hospital of Tongji University, Shanghai, People's Republic of China

Full list of author information is available at the end of the article
}

analyzed in parallel by spatial transcriptomics (ST, $10 \times$ genomics) (Fig. 1a) [4, 5]. By analyzing the transcriptomes, we detected 9 distinct cell clusters in the BC-A tumors by using canonical lineage markers (Fig. 1b). The epithelial cells were further re-clustered, and 6 subclusters were identified, respectively (Fig. 1c). The copy number variation $(\mathrm{CNV})$ profiles of the $\mathrm{BC}$-A tumor cells show a substantial degree of heterogeneity, suggesting that the origin of these subclusters was variable. Chr8q gain in genomic unstable luminal-A cells was found in the BC-A profile (Additional file 1: Figure S1A). Furthermore, the malignant subclusters were annotated with cluster-specific genes mainly including the basal subtype $\left(E G F R^{+} K I T^{+}\right.$basal cells and $E G F R^{+} T{ }^{2} 3^{+}$normallike cells) and luminal subtype (cycling luminal-B cells, $E R B B 4^{+}$luminal-A cells, $E R B B 4^{+} A R E G^{+}$luminal-A cells and genomic unstable luminal-A cells) in the BC-A sample (Fig. 1c-e) (Additional file 2: Result S1). To characterize the functional features of these distinct cancer clusters, we dissected their differentially expressed genes. Cycling luminal-B (LumB) cells were uniquely enriched for hallmarks of the cell cycle and proliferation (e.g., E2F

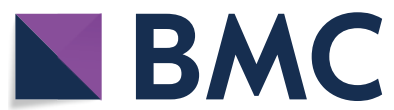

(c) The Author(s) 2022. Open Access This article is licensed under a Creative Commons Attribution 4.0 International License, which permits use, sharing, adaptation, distribution and reproduction in any medium or format, as long as you give appropriate credit to the original author(s) and the source, provide a link to the Creative Commons licence, and indicate if changes were made. The images or other third party material in this article are included in the article's Creative Commons licence, unless indicated otherwise in a credit line to the material. If material is not included in the article's Creative Commons licence and your intended use is not permitted by statutory regulation or exceeds the permitted use, you will need to obtain permission directly from the copyright holder. To view a copy of this licence, visit http://creativecommons.org/licenses/by/4.0/. The Creative Commons Public Domain Dedication waiver (http://creativeco mmons.org/publicdomain/zero/1.0/) applies to the data made available in this article, unless otherwise stated in a credit line to the data. 


\section{(See figure on next page.)}

Fig. 1 snRNA-seq analysis of tumor from sample BC-A. a Schematic of the single-nucleus RNA-seq and ST experiment and analysis. b UMAP visualization of 4,093 nuclei from BC-A tumor analyzed by snRNA-seq showing nine major cell types. c UMAP visualization of inferred epithelial cells from BC-A tumor analyzed by snRNA-seq. Clusters are colored and labeled according to their inferred cell subtypes. $\mathbf{d}$ Feature plot of subcluster-specific marker genes in epithelial cells. e Heatmap of differentially expressed genes in each epithelial subcluster. The color bars above the heatmap reflects the subcluster PAM50 subtype estimated by 'pseudobulk' $\mathbf{f}$ Heatmap showing the pathway enrichment of each epithelial subcluster using MSigDB HALLMARK gene sets. Mean score of GSVA was z-score transformed. $\mathbf{g}$ Heatmap of the area under the curve (AUC) scores of TF motifs estimated per cell by SCENIC. $\mathbf{h}$ Differentiation trajectories of epithelial cells by Monocle 2. $\mathbf{i}$ Circos plot showing the interactions between ligands and receptors across cell types

and MYC TARGETS), and this cluster exhibited a high metabolic state with the activation of glycatabolism, fatty acid and cholesterol metabolism potentially regulated by $\mathrm{PI} 3 \mathrm{~K} / \mathrm{AKT} / \mathrm{mTOR}$ signaling, hypoxia and MYC (Fig. 1f, Additional file 1: Figure S1B-C). In contrast, genomic unstable luminal-A (LumA) cells only showed an activated KRAS-downregulated signaling (Fig. 1f). Next, we asked whether transcription factors (TFs) contribute to the phenotypic state of these subpopulations (Fig. 1g). First, GATA3, a key mediator during the differentiation from luminal progenitor to mature luminal cells, was active in these luminal clusters. AP-1 transcription factor complexes, including JUN, JUNB, JUND, FOS and FOSB, were enriched in $E R B B 4^{+} A R E G^{+}$LumA, genomic unstable LumA, $E G F R^{+} K I T^{+}$basal and cycling LumB clusters. Likewise, $E R B B 4^{+} A R E G^{+}$LumA cluster also highly expressed SREBF1/2 which are crucial for controlling cholesterol homeostasis [6]. Pseudotime analysis revealed trajectories of luminal and basal/myoepithelial lineages (Fig. 1h). The six major cell clusters were corroborated by typical mammary lineage marker genes for basal progenitor/myoepithelium (e.g., MYLK and ACTA2), mature luminal cells (e.g., ESR1, FOXA1, PGR and AFF3) and luminal progenitor cells (e.g., EZH2) (Additional file 1: Figure S3C-D) [7]. Moreover, the myoepithelial cells stemmed from basal progenitor cells, while luminal progenitor cells gradually differentiated into mature luminal cells (Fig. 1h). Interestingly, a cluster of luminal cells expressed certain level of basal-like genes, suggesting that they may emerge as a dedifferentiated state or represent invasiveness. EGFR ${ }^{+} \mathrm{TP}^{+} 3^{+}$normal-like subtype was fully distributed in the left arm, while cycling LumB cluster was completely situated in the arm of the luminal progenitor (Fig. 1h). Then, we investigated the interactions between ligands and receptors across all neoplastic populations. Interestingly, $E R B B 4^{+} A R E G^{+}$LumA cells secreted increasing EGF and EGF-like cytokine-like AREG, which could combine with EGF receptors such as EGFR and ERBB4 (Fig. 1i, Additional file 1: Figure S1D) (Additional file 2: Result S2).

Subsequently, we sought to annotate the intratumoral heterogeneity by integrating the snRNA-seq and ST datasets [5]. The ST dataset was classified into six main areas: the luminal region, basal region, the interfacing area between the luminal region and basal region, stroma and infiltrating lymphocyte areas based on the principal component scores across all ST spots (Fig. 2a, b, Additional file 1: S5C). We found that these luminal subtypes were principally assembled in the luminal region and the interfacing area except that the cycling LumB cells were scattered throughout the tissue. Interestingly, the $E R B B 4^{+}$LumA type scarcely existed in the interfacing area (Fig. 2a, b, Additional file 1: S5B). Finally, the spatial division of the primary nontumor cells was identified in the ST dataset, showing that neutrophils were enriched in the luminal region, while B cells principally infiltrated into the basal region (Fig. 2c) (Additional file 2: Result S3).

We deconvoluted breast cancer profiles in the METABRIC cohort [8]. Based on the defined gene signature, all of cases were divided into three discriminated subgroups (DSGs). And DSG2, representing the HER2-overexpressing subtypes based on PAM50 was designated the genomic unstable LumA subtypes (Fig. 2d). For survival analysis, patients representing the DSG2 subtype were associated with the worst survival (Fig. 2e). Through examining public datasets of patients treated with chemotherapy[9], the proportions of the $E R B B 4^{+}$ LumA cells or $E G F R^{+} T P 63^{+}$normal-like cells were associated with poor response to neoadjuvant chemotherapy (NAC) while the ratio of the cycling LumB cluster significantly increased in patients who response to NAC (Fig. 2f) (Additional file 2: Result S4, Additional file 3).

In summary, we have provided a comprehensive approach to depict the heterogeneity and the architecture of breast cancer. Our study could provide novel insights 
A

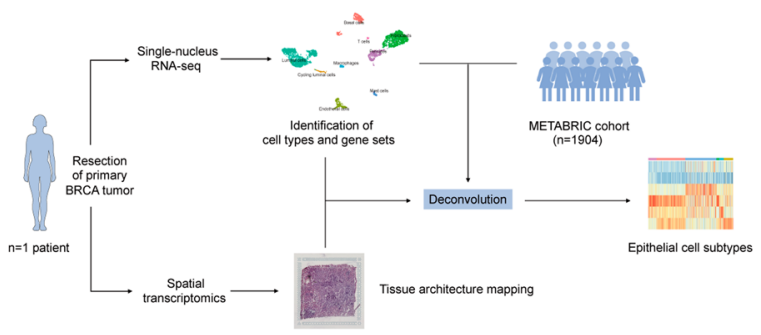

B

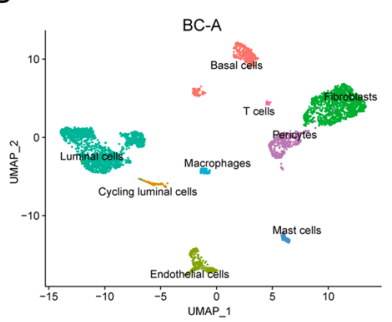

C

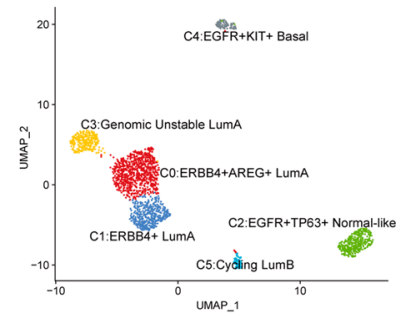

$\mathrm{F}$

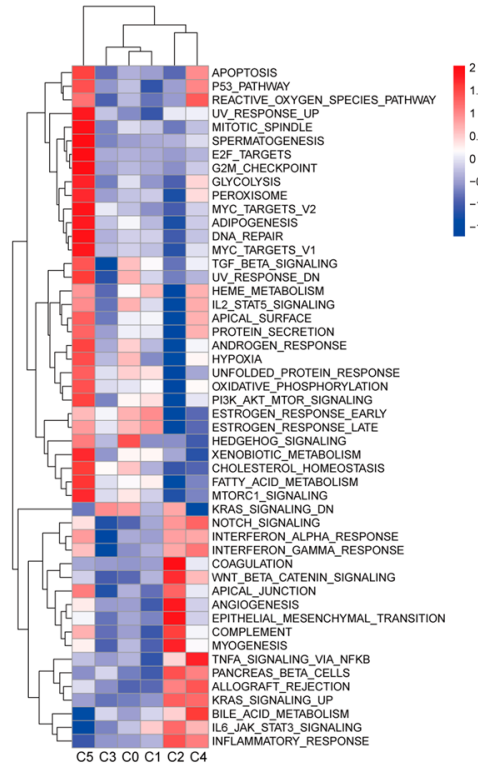

D
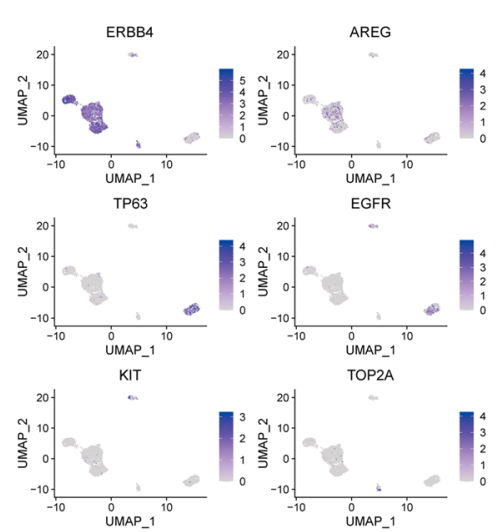

G
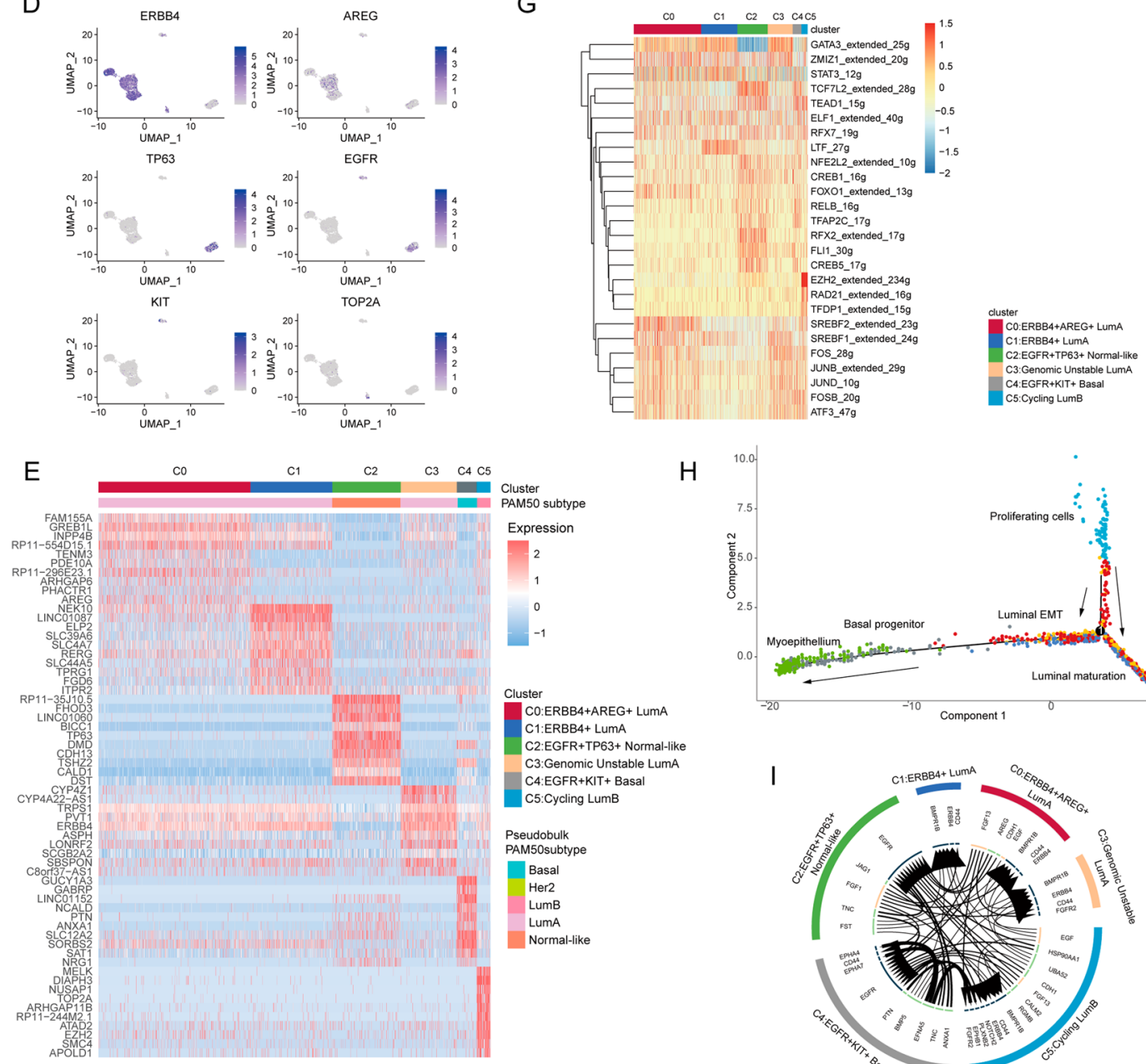

$\mathrm{H}$

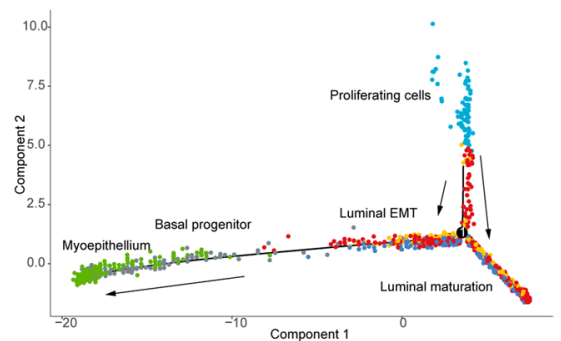

C0:ERBB4+AREG+ LumA C1:ERBB4+ LumA

C2:EGFR+TP63+ Normal-like

C3:Genomic Unstable LumA

C4:EGFR+KIT+ Basa

Pseudobulk
PAM50subtype

Basal
Her2

LumB

LumA

Fig. 1 (See legend on previous page.) 


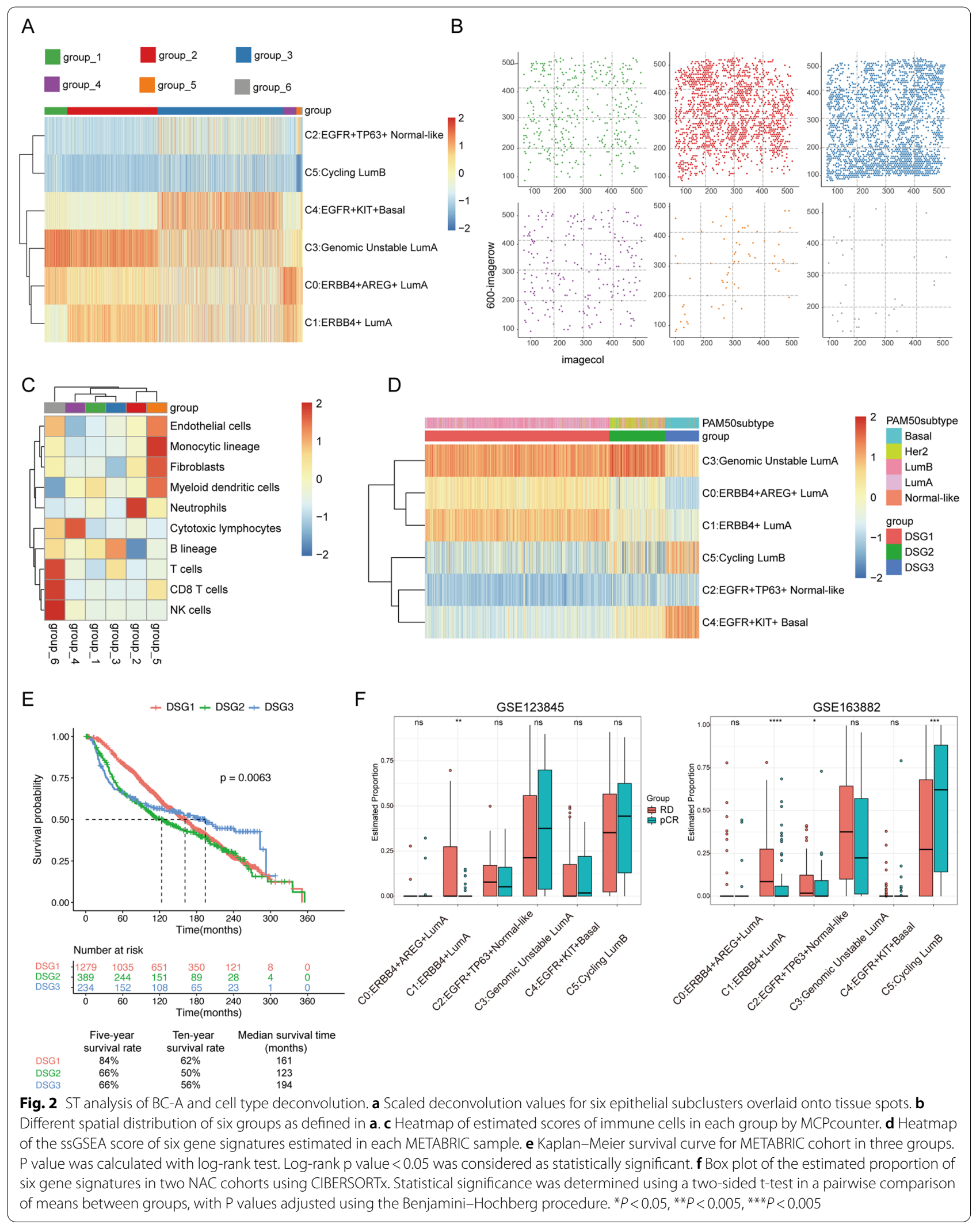


into the ecosystem of breast cancer and novel therapeutic strategies.

\begin{abstract}
Abbreviations
snRNA-seq: Single-nucleus RNA sequencing; ST: Spatial transcriptomics; BC: Breast cancer; CNV: Copy number variation; LumA: Luminal-A; LumB: LuminalB; AREG: Amphiregulin; EGF: Epidermal growth factor; TGFa: Transforming growth factor alpha; EMT: Epithelial-mesenchymal transition; PTN: Pleiotrophin; EFNA5: Ephrin; TFs: Transcription factors; LTF: Lactotransferrin; ECM: Extracellular matrix; H\&E: Hematoxylin and eosin; DCIS: Ductal carcinoma in situ; IHC: Immunohistochemistry; ER: Estrogen receptor; PR: Progesterone receptor; DSG: Discriminated subgroups; NAC: Neoadjuvant chemotherapy; pCR: Pathologic complete response; RD: Residual disease.
\end{abstract}

\section{Supplementary Information}

The online version contains supplementary material available at https://doi. org/10.1186/s13045-022-01236-0.

\section{Additional file 1. Figures.}

Additional file 2. Results.

Additional file 3. Materials and methods.

\section{Acknowledgements}

We thank a professional language editing by American Journal Experts.

\section{Authors' contributions}

QW contributed to conceptualization; XYM and JW were involved in methodology; SQL, ZJG and JW analyzed the data; QW, SQL, ZJG and XYM wrote and prepared the original draft; SQL and ZJG took part in writing, reviewing and editing; HMZ conducted the supervision; BL participated in project administration; and SS acquired the funding. All authors read and approved the final manuscript.

\section{Funding}

This work was supported by a National Natural Science Foundation of China (NSFC) grant (Grant NO: 81471781) to Prof. Sheng-Rong Sun and (Grant NO: 81903166) to Dr. Si Sun. This work was also supported by a Hubei Province health and family planning scientific research project to Dr. Si Sun (Grant No. WJ2019Q044) and a project (WJ2019Q053) to Dr. Hong-Mei Zheng. And this work was supported by a Fundamental Research Funds for the Central Universities of China grant (Grant NO: 413000480) to Dr. Qi Wu. Finally, this work was supported by the Natural Science Foundation of Hubei (Grant No. 2018CKB916) to Dr. Si Sun and a project (2020CFB874) to Dr. Hong-Mei Zheng. Dr. Xiang-Yu Meng was supported by a fellowship from ITMO Cancer AVIESAN within the framework of Cancer Plan.

\section{Availability of data and materials}

The datasets used and/or analyzed during the current study are available from the corresponding author on reasonable request.

\section{Declarations}

Ethics approval and consent to participate Not applicable.

\section{Consent for publication}

Not applicable.

\section{Competing interests}

The authors declare that they have no competing interests.

\section{Author details}

${ }^{1}$ Department of Breast and Thyroid Surgery, Renmin Hospital of Wuhan University, Wuhan, Hubei, People's Republic of China. ${ }^{2}$ Department of Pathology, Renmin Hospital of Wuhan University, Wuhan, Hubei, People's Republic of China. ${ }^{3}$ Department of Breast Surgery, Hubei Cancer Hospital, Tongji Medical College, Huazhong University of Science and Technology, Wuhan, Hubei, People's Republic of China. ${ }^{4}$ Department of Clinical Laboratory, Renmin Hospital of Wuhan University, Hubei Province, 238 Ziyang Road, Wuhan 430060, People's Republic of China. ${ }^{5}$ Center for Single-Cell Omics and Tumor Liquid Biopsy, Zhongnan Hospital of Wuhan University, Wuhan, Hubei, People's Republic of China. ${ }^{6}$ Tongji University Cancer Center, Tenth People's Hospital of Tongji University, Shanghai, People's Republic of China.

Received: 27 December 2021 Accepted: 11 February 2022

Published online: 03 March 2022

\section{References}

1. Moncada R, Barkley D, Wagner F, Chiodin M, Devlin JC, Baron M, Hajdu $\mathrm{CH}$, Simeone DM, Yanai I. Integrating microarray-based spatial transcriptomics and single-cell RNA-seq reveals tissue architecture in pancreatic ductal adenocarcinomas. Nat Biotechnol. 2020;38(3):333-42.

2. Lei Y, Tang R, Xu J, Wang W, Zhang B, Liu J, Yu X, Shi S. Applications of single-cell sequencing in cancer research: progress and perspectives. J Hematol Oncol. 2021;14(1):91.

3. Wu SZ, Al-Eryani G, Roden DL, Junankar S, Harvey K, Andersson A, Thennavan A, Wang C, Torpy JR, Bartonicek N, Wang T, Larsson L, Kaczorowsk D, Weisenfeld NI, Uytingco CR, Chew JG, Bent ZW, Chan CL, Gnanasambandapillai V, Dutertre CA, Gluch L, Hui MN, Beith J, Parker A, Robbins E, Segara D, Cooper C, Mak C, Chan B, Warrier S, Ginhoux F, Millar E, Powell JE, Williams SR, Liu XS, O’Toole S, Lim E, Lundeberg J, Perou CM, Swarbrick A. A single-cell and spatially resolved atlas of human breast cancers. Nat Genet. 2021;53(9):1334-47.

4. Stahl PL, Salmen F, Vickovic S, Lundmark A, Navarro JF, Magnusson J, Giacomello S, Asp M, Westholm JO, Huss M, Mollbrink A, Linnarsson S, Codeluppi S, Borg A, Ponten F, Costea PI, Sahlen P, Mulder J, Bergmann $\mathrm{O}$, Lundeberg J, Frisen J. Visualization and analysis of gene expression in tissue sections by spatial transcriptomics. Science. 2016;353(6294):78-82.

5. Fu T, Dai LJ, Wu SY, Xiao Y, Ma D, Jiang YZ, Shao ZM. Spatial architecture of the immune microenvironment orchestrates tumor immunity and therapeutic response. J Hematol Oncol. 2021;14(1):98.

6. Xu D, Wang Z, Xia Y, Shao F, Xia W, Wei Y, Li X, Qian X, Lee JH, Du L, Zheng Y, Lv G, Leu JS, Wang H, Xing D, Liang T, Hung MC, Lu Z. The gluconeogenic enzyme PCK1 phosphorylates INSIG1/2 for lipogenesis. Nature. 2020;580(7804):530-5.

7. Hu L, Su L, Cheng H, Mo C, Ouyang T, Li J, Wang T, Fan Z, Fan T, Lin B, Zhang J, Xie Y. Single-Cell RNA Sequencing Reveals the Cellular Origin and Evolution of Breast Cancer in BRCA1 Mutation Carriers. Cancer Res. 2021;81(10):2600-11.

8. C. Curtis, S.P. Shah, S.F. Chin, G. Turashvili, O.M. Rueda, M.J. Dunning, D. Speed, A.G. Lynch, S. Samarajiwa, Y. Yuan, S. Graf, G. Ha, G. Haffari, A. Bashashati, R. Russell, S. McKinney, M. Group, A. Langerod, A. Green, E. Provenzano, G. Wishart, S. Pinder, P. Watson, F. Markowetz, L. Murphy, I. Ellis, A. Purushotham, A.L. Borresen-Dale, J.D. Brenton, S. Tavare, C. Caldas, S. Aparicio. The genomic and transcriptomic architecture of 2,000 breast tumours reveals novel subgroups. Nature. 2012;486(7403):346-52.

9. Park YH, Lal S, Lee JE, Choi YL, Wen J, Ram S, Ding Y, Lee SH, Powell E, Lee SK, Yu JH, Ching KA, Nam JY, Kim SW, Nam SJ, Kim JY, Cho SY, Park S, Kim J, Hwang S, Kim YJ, Bonato V, Fernandez D, Deng S, Wang S, Shin H, Kang ES, Park WY, Rejto PA, Bienkowska J, Kan Z. Chemotherapy induces dynamic immune responses in breast cancers that impact treatment outcome. Nat Commun. 2020;11(1):6175.

\section{Publisher's Note}

Springer Nature remains neutral with regard to jurisdictional claims in published maps and institutional affiliations. 